\title{
Experimental Study of a Phase Modulator Using an Active Interferometric Device
}

\author{
Rogério Dionísio ${ }^{\# 1}$, Cláudia Reis ${ }^{\# 2}$, Paulo André ${ }^{\# 3}$, Rogério Nogueira ${ }^{\# 4}$ and António Teixeira ${ }^{\#+5}$ \\ * Instituto de Telecomunicações \\ Campus Universitário de Santiago, 3810-193 Aveiro, Portugal \\ 1,2,3,4 \{rdionisio, creis, pandre, rnogueira\}@av.it.pt \\ * Escola Superior de Tecnologia do Instituto Politécnico de Castelo Branco \\ Avenida do Empresário, 6000-767 Castelo Branco, Portugal \\ ${ }^{+}$Departamento de Electrónica, Telecomunicações e Informática, Universidade de Aveiro \\ Campus Universitário de Santiago, 3810-193 Aveiro, Portugal \\ ${ }^{5}$ teixeira@ua.pt
}

\begin{abstract}
A novel architecture for an optical phase modulator is presented and experimentally demonstrated. This approach relies on a commercially available integrated MachZehnder interferometer structure with Semiconductor Optical Amplifiers (MZI-SOA) and it is based in cross-phase modulation effect (XPM). The feasibility of the proposed optical phase modulator is experimentally investigated using different scenarios of input power and bit rates.
\end{abstract}

\section{INTRODUCTION}

In the past few years, the data volume of communication networks increased dramatically, so there is a need for finding fast optical transmission techniques, along with equipment with low power consumption and integration facilities. Among those techniques, optical phase modulation is an option that allow greater transmission distances in both digital and analog transmission systems [1].

Phase modulation generates signals of 1 and 0 by changing the phase of light while allowing it to be in the on position. As opposed to intensity modulation, phase modulation has superior bandwidth efficiency and is not easily affected by signal distortions caused by transmission fibers and relay nodes.

Several optical techniques have been proposed to implement optical phase modulators. In [2] the scheme proposed is a phase modulation based on frequency shifters, which consists of an acousto-optic modulator (AOM), followed by a fiber. In [3] and [4] $\mathrm{LiNbO} 3$ waveguide-based phase modulator and gain-transparent semiconductor optical amplifier (GT-SOA) are used, respectively, as optical phase modulators. In [5], a Highly Non-Linear Fiber (HNLF) is used as the optical medium to phase modulate a continuous wave (CW) laser.

In this paper, we propose an optical phase modulator based on the XPM effect [6] using a MZI-SOA. To the authors' knowledge, this is the first demonstration of optical phase modulation using both interferometric arms of a MZI-SOA. This technique can be used in multi-level modulation signals generation [7] as well in OCDMA transmission systems [8].

\section{SYSTEM DESCRIPTION}

\section{A. Principle of Operation}

Fig. 1(a) is a schematic diagram of the MZI-SOA. We use a commercial hybrid-integrated device consisting of a passive, planar silica balanced Mach-Zehnder interferometer with nonlinear Semiconductor Optical Amplifiers and phase shifters assembled in each interferometer arm.

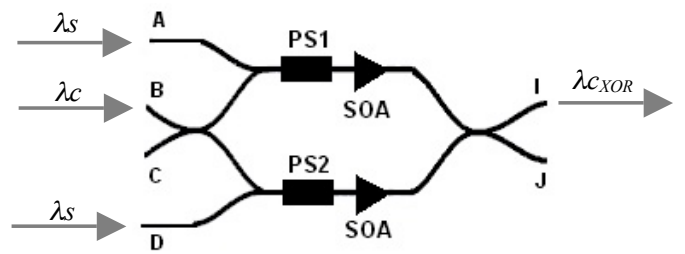

(a) Schematic diagram of the MZI-SOA

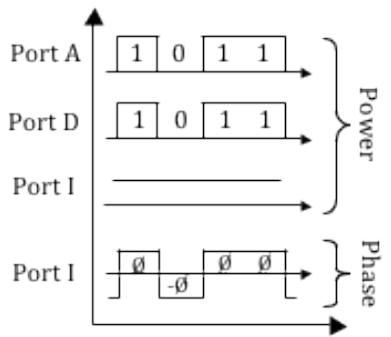

(b) Principle of operation

\begin{tabular}{|c|c|c|}
\hline port A & port D & $\begin{array}{c}\text { port I } \\
\text { (XOR) }\end{array}$ \\
\hline 0 & 0 & 0 \\
\hline 0 & 1 & 1 \\
\hline 1 & 0 & 1 \\
\hline 1 & 1 & 0 \\
\hline
\end{tabular}

(c) XOR truth table
Fig. 1. Schematic diagram of a MZI-SOA, principle of operation and XOR truth table.

Two input data streams at the same wavelength $\lambda s$ are coupled into ports $\mathrm{A}$ and D of the MZI-SOA, while a CW light at $\lambda c$ is coupled into port $\mathrm{B}$. Inside the interferometer, data signals are launched into the two SOAs where they modulate the carrier density and also the refractive index [9]. The intensity variations of the input optical signals cause a phase modulation of the control $\mathrm{CW}$ signal propagating through the SOAs. If both data signals are time synchronized, 
the CW light from the two SOAs interferes destructively. According to the XOR operation in Fig. 1(c), no pulse is observed at the output port I of the interferometer [10]. However, the phase $\phi$ of $\lambda c_{X O R}$ will vary in accordance to the input pattern, as depicted Fig. 1(b) [11].

\section{B. Experimental Setup}

Fig. 2 illustrates the experimental setup of the proposed optical phase modulator. It consists of an external cavity laser peaking at $1549.32 \mathrm{~nm}$, followed by a polarization controller and a Mach-Zehnder external modulator. The NRZ data signal generated by a serial BERT (Agilent N4901B) is then amplified by an EDFA (IPG-EAD-500-C3-W) and split into two equal parts using a $3 \mathrm{~dB}$ coupler. Both signals are synchronized using optical delay lines. Polarization controllers are included at ports A and D of a MZI-SOA (CIP 40G-2R2-ORP), in order to optimize the destructive output signal at port I.

The control signal, a CW light beam lasing at $1546.12 \mathrm{~nm}$, is launched into port B of the MZI-SOA in a co-propagating direction with the data signals.

Finally, the control signal is recovered at port I, using a filter with a $40 \mathrm{GHz}$ bandwidth (X-tract Net Test). We use two measurement instruments to analyze the output signal: an oscilloscope (Agilent 86100A), connected through a PIN photodiode (HP-11982A), and an optical complex spectrum analyzer (APEX AP 2441A) to gather phase and power information of the output signal, for time domain characterization.

\section{EXPERIMENTAL RESULTS AND DISCUSSION}

In order to validate the feasibility of MZI-SOA based phase modulators, experiments at 2.5 and $10 \mathrm{~Gb} / \mathrm{s}$ were carried out with the same experimental setup.
An average extinction ratio (ER) of $11.3 \mathrm{~dB}$ and a mean power of $2.5 \mathrm{dBm}$ were measured for data signals launched into ports $\mathrm{A}$ and $\mathrm{D}$ of the MZI-SOA.

The bias current (Isoa) of both SOAs were varied simultaneously, from 150 to $300 \mathrm{~mA}$ for $2.5 \mathrm{~Gb} / \mathrm{s}$ and from 150 to $400 \mathrm{~mA}$ for $10 \mathrm{~Gb} / \mathrm{s}$. For each bias current, the mean power of the control signal $\left(\mathrm{P}_{\mathrm{CW}}\right)$ was increased from -6 to $2 \mathrm{dBm}$.

The voltage applied to the phase shifters was adjusted in order to maximize the destructive interference at output port I.

\section{A. Phase modulator experiments at $2.5 \mathrm{~Gb} / \mathrm{s}$}

Due to limitations imposed by the OCSA, the length's sequence at $2.5 \mathrm{~Gb} / \mathrm{s}$ was restricted to 4 bits [12].

Fig. 3(c) shows the bit pattern launched at the interferometric ports (A and D) of the MZI-SOA. Fig. 3(d) illustrate the output signal at port I for bias current at $250 \mathrm{~mA}$ and control signal at $-4 \mathrm{dBm}$. The phase shift related to different logic levels is well defined and the output power signal is inverted when compared with the input data signals.

In Fig. 3(a), phase span is plotted as a function of $\mathrm{P}_{\mathrm{CW}}$ for several bias currents. The results show that they increase as the bias current is raised. Mean values vary between $35^{\circ}$ and $50^{\circ}$.

It can be observed in Fig. 3(b) that the mean output power is also proportional to the increase of Isoa and $\mathrm{P}_{\mathrm{CW}}$ since the SOAs gain is not saturated.

\section{B. Phase modulator experiments at $10 \mathrm{~Gb} / \mathrm{s}$}

The proposed optical phase modulator was also evaluated at $10 \mathrm{~Gb} / \mathrm{s}$. The tests were performed using data sequences with 16 bits [12].

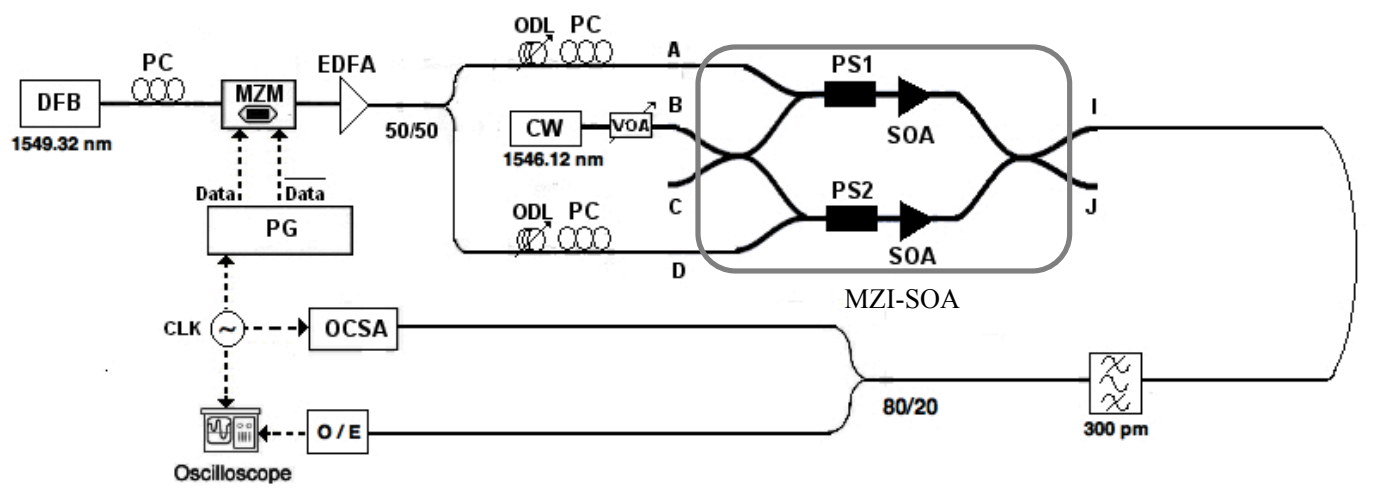

Fig. 2. Experimental setup of the optical phase modulator. DFB: distributed-feedback laser; PC: polarization controller; MZM: Mach-Zehnder modulator; EDFA: Erbium Doped Fiber Amplifier; ODL: optical delay line; CW: continuous wave laser; VOA: variable optical attenuator; SOA: semiconductor optical amplifier; PS1, 2: phase shifters; OCSA: optical complex spectrum analyzer; PG: pattern generator; O/E: PIN photodiode. Solid lines represent fiber-optic paths and dashed lines indicate electronic connections. 


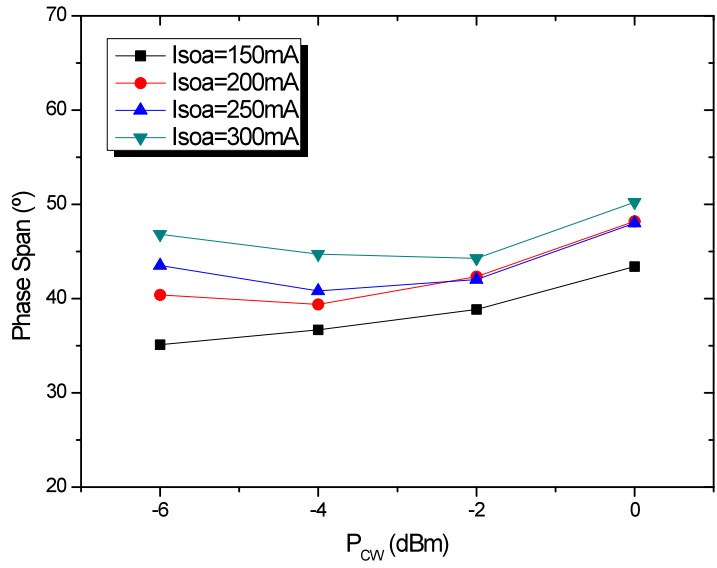

(a) Output phase span

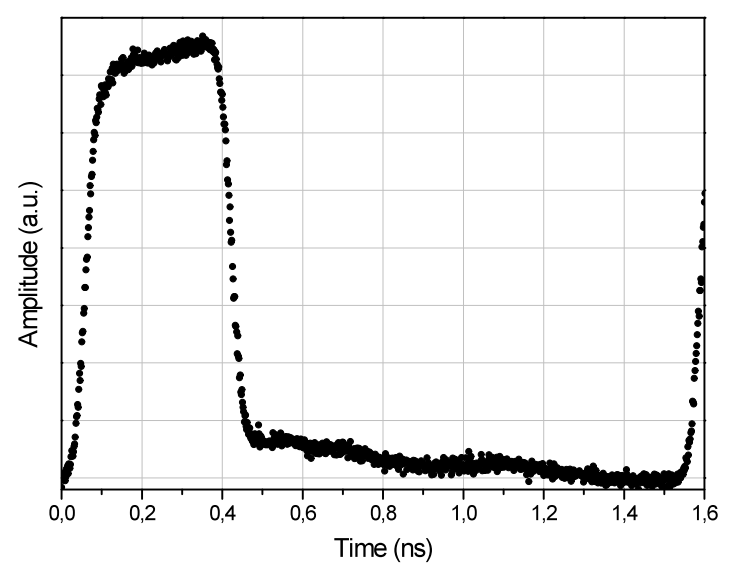

(c) Input signal with sequence "1000"

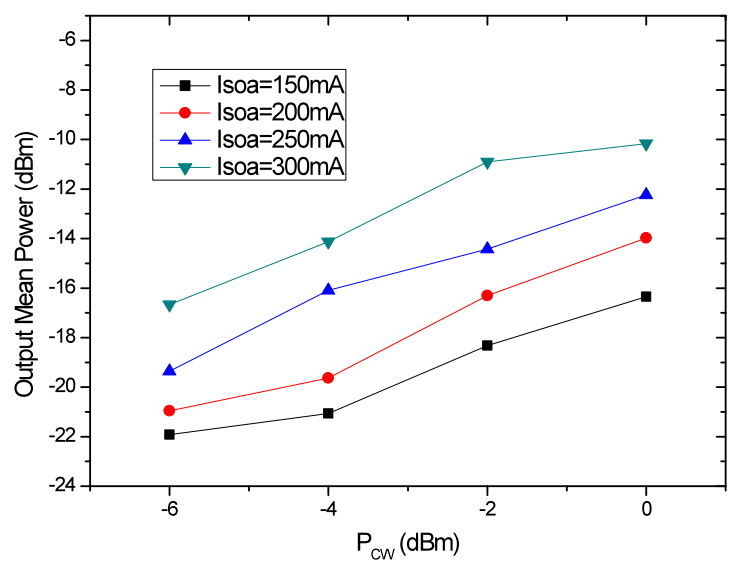

(b) Output mean power

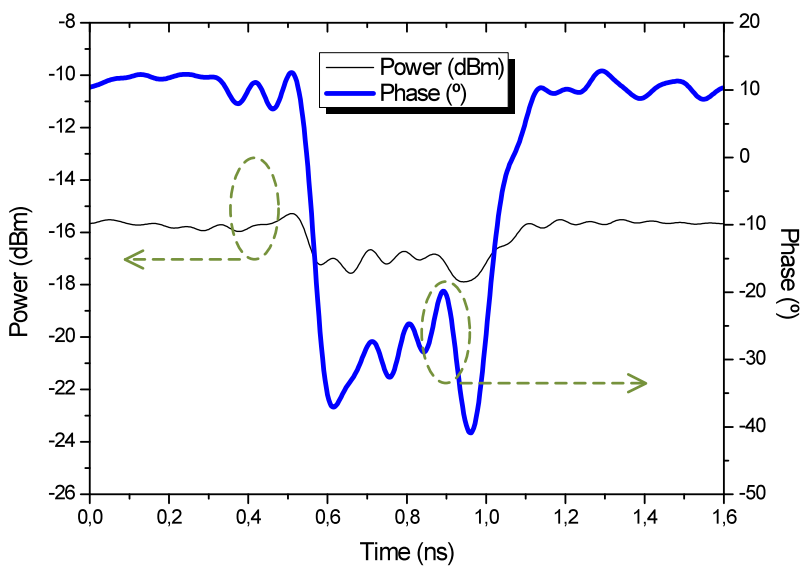

(d) Phase and power output for Isoa $=250 \mathrm{~mA}$ and $\mathrm{P}_{\mathrm{CW}}=-4 \mathrm{dBm}$

Fig. 3. Experimental results at $2.5 \mathrm{~Gb} / \mathrm{s}$

Fig. 4(c) shows the bit pattern coupled at the input ports (A and D) of the MZI-SOA. The resulting output signal with bias current at $150 \mathrm{~mA}$ and control signal at $0 \mathrm{dBm}$ is depicted in Fig. 4(d). Output power fluctuations are mainly due to noise. As with $2.5 \mathrm{~Gb} / \mathrm{s}$ experiments, phase shifts are inverted when compared with data signal logic levels. However, due to the dynamics of the SOA and the carrier recovery time, output phase levels are less pronounced at $10 \mathrm{~Gb} / \mathrm{s}$ when fast variations occurs at the MZI-SOA data inputs. Phase constellations diagrams in Fig. 4(a) shows that phase logic levels are evenly defined when the bias current is increased.

As it can be seen in Fig. 4(a) and Fig 4(b), we obtain higher values of phase span and output mean power, respectively, by increasing the bias current.

For $\mathrm{P}_{\mathrm{CW}}$ ranging from -6 to $0 \mathrm{dBm}$, SOAs are in linear amplification regime. In this case, the mean values of the phase span vary between $70^{\circ}$ and $170^{\circ}$. For $\mathrm{P}_{\mathrm{CW}}$ above $0 \mathrm{dBm}$, the SOAs saturates, which reduces phase span values and output mean powers.

\section{CONCLUSION}

In this paper, a new way of performing optical phase modulation has been presented. We assess the impact of SOAs bias current and input $\mathrm{CW}$ power on the phase of the destructive output of a MZI-SOA.

We observed that an increase of the bias current produces higher values of phase spans and output mean powers. However, SOAs gain saturation has an opposite effect on the output signal.

The experimental results demonstrate the feasibility of an MZI-SOA device as an optical phase modulator. Other options for phase modulation exist, using a single waveguide embedded in an electro-optical substrate $\left(\mathrm{LiNbO}_{3}\right)$ or using the principle of interference with two waveguides to cause also an amplitude modulation of the optical signal (as in a MZM). However, those methods introduce insertion losses. Using an MZI-SOA, not only the losses are compensated, but also the optical power can be increased by the SOAs. 


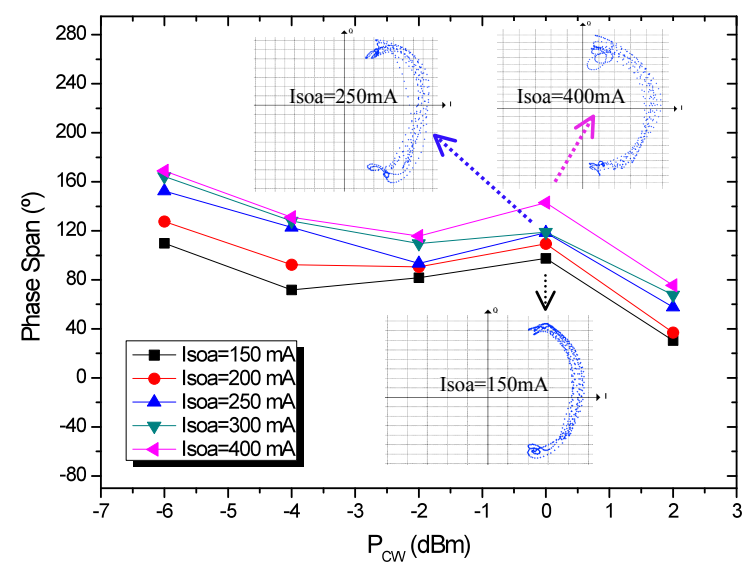

(a) Output phase span

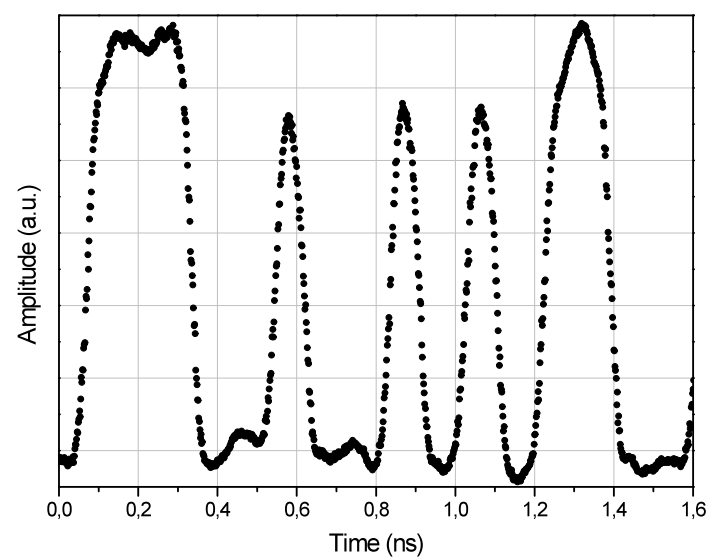

(c) Input sequence "1110010010101100"

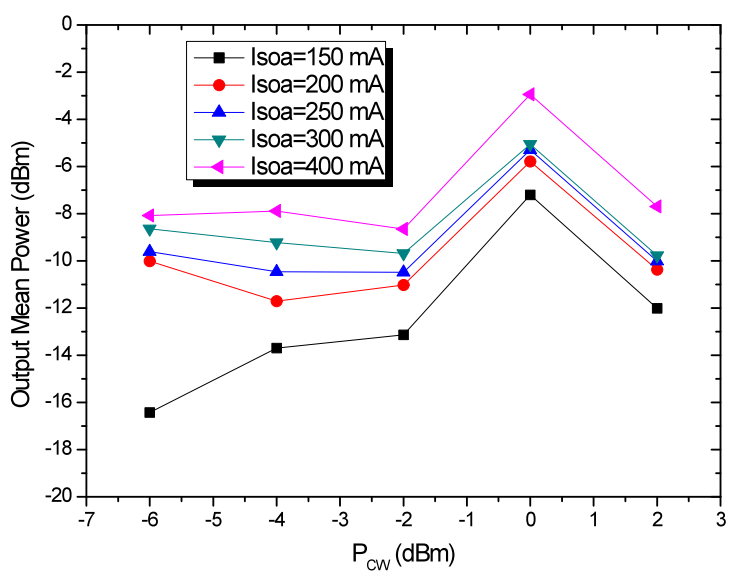

(b) Output mean power

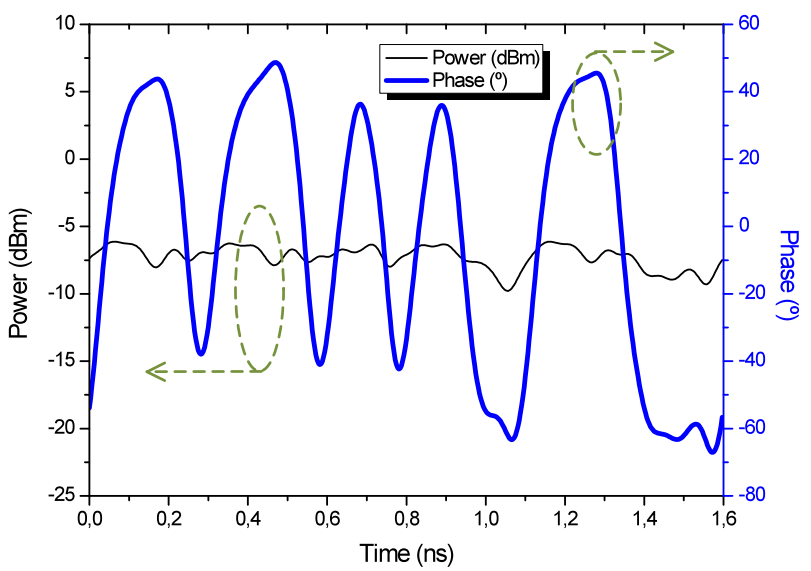

(d) Phase and power output for Isoa $=150 \mathrm{~mA}$ and $\mathrm{P}_{\mathrm{CW}}=0 \mathrm{dBm}$

Fig. 4. Experimental results at $10 \mathrm{~Gb} / \mathrm{s}$

Future research and application include high-level modulation formats (m-QSK, m-QAM), using several MZI-SOAs in serial or parallel configuration, within access to metro or long-haul connection nodes, making at the same time amplification and conversion of amplitude modulated signals to high level advanced modulation formats.

\section{ACKNOWLEDGMENT}

The authors greatly acknowledge Networks of Excellence EURO-FOS, BONE and also PANORAMA (ADI 2009/003144) and THRONE (PTDC/EEA-TEL/66840/2006) projects.

\section{REFERENCES}

[1] M. N. Sysak, et al., "Characterization of third order distortion in InGaAsP optical phase modulator monolithically integrated with balanced UTC photodetector," 2006 Ieee Leos Annual Meeting Conference Proceedings, Vols 1 and 2, pp. 338-339, 2006.
B. Qi, et al., "Polarization insensitive phase modulator for quantum cryptosystems," Opt. Express, vol. 14, pp. 4264-4269, 2006.

C. Langrock, et al., "Highly efficient single-photon detection at communication wavelengths by use of upconversion in reverseproton-exchanged periodically poled $\mathrm{LiNbO3}$ waveguides," Opt. Lett., vol. 30, pp. 1725-1727, 2005.

W. Hong, et al., "Simulation and analysis of gain-transparent SOA used as optical phase-modulator in DPSK applications," Wuhan, China, 2007, pp. 67822L-12.

V. Marembert, et al., "Investigations of fiber Kerr switch: nonlinear phase shift measurements and optical time-division demultiplexing of $320 \mathrm{Gbit} / \mathrm{s}$ DPSK signals," in Lasers and Electro-Optics, 2005. (CLEO). Conference on, 2005, pp. 14321434 Vol. 2.

T. T. Ng, et al., "Characterization of XGM and XPM in a SOAMZI using a Linear Frequency Resolved Gating Technique," in Lasers and Electro-Optics Society, 2007. LEOS 2007. The 20th Annual Meeting of the IEEE, 2007, pp. 656-657.

H. Keang-Po and C. Han-Wei, "Generation of arbitrary quadrature signals using one dual-drive Modulator," Lightwave Technology, Journal of, vol. 23, pp. 764-770, 2005.

P. Teixeira, et al., "Automatic apodization profiling of super structured fiber Bragg gratings for OCDMA coding applications," 
2008 Conference on Optical Fiber Communication/National Fiber Optic Engineers Conference, Vols 1-8, pp. 85-87, 2008.

[9] T. Fjelde, et al., "Demonstration of $20 \mathrm{Gbit} / \mathrm{s}$ all-optical logic XOR in integrated SOA-based interferometric wavelength converter," Electronics Letters, vol. 36, pp. 1863-1864, 2000. R. Vilar, et al., "All-optical DGD monitor for packet-switched
$[11]$ networks based on an integrated active Mach-Zehnder interferometer operating as logic XOR gate," Optics Communications, vol. 281, pp. 5330-5334, 2008.
S. C. Cao and J. C. Cartledge, "Measurement-based method for characterizing the intensity and phase modulation properties of SOA-MZI wavelength converters," Photonics Technology Letters, IEEE, vol. 14, pp. 1578-1580, 2002.

[12] (2009, November 10). APEX Optical Spectrum Analyzer AP2041B [pdf]. Available: http://www.apex-t.com/pdf/ap2040a.pdf 